\title{
A note on random minimum length spanning trees
}

\author{
Alan Frieze* Miklós Ruszinkó ${ }^{\dagger}$ \\ Lubos Thoma ${ }^{\ddagger}$ \\ Department of Mathematical Sciences \\ Carnegie Mellon University \\ Pittsburgh PA15213, USA \\ alan@random.math. cmu.edu, \\ ruszinko@lutra.sztaki.hu, \\ thoma@qwes.math. cmu.edu
}

Submitted June 28, 2000, Accepted August 11, 2000

\begin{abstract}
Consider a connected $r$-regular $n$-vertex graph $G$ with random independent edge lengths, each uniformly distributed on $[0,1]$. Let $m s t(G)$ be the expected length of a minimum spanning tree. We show in this paper that if $G$ is sufficiently highly edge connected then the expected length of a minimum spanning tree is $\sim \frac{n}{r} \zeta(3)$. If we omit the edge connectivity condition, then it is at most $\sim \frac{n}{r}(\zeta(3)+1)$.
\end{abstract}

\section{Introduction}

Given a connected simple graph $G=(V, E)$ with edge lengths $\mathbf{x}=\left(x_{e}: e \in E\right)$, let $m s t(G, \mathbf{x})$ denote the minimum length of a spanning tree. When $\mathbf{X}=\left(X_{e}: e \in E\right)$ is a family of independent random variables, each uniformly distributed on the interval $[0,1]$, denote the expected value $\mathbf{E}(m s t(G, \mathbf{X}))$ by $m s t(G)$. Consider the complete graph $K_{n}$. It is known (see [2]) that, as $n \rightarrow \infty, \operatorname{mst}\left(K_{n}\right) \rightarrow \zeta(3)$. Here $\zeta(3)=\sum_{j=1}^{\infty} j^{-3} \sim 1.202$. Beveridge, Frieze and McDiarmid [1] proved two theorems that together generalise the previous results of [2], [3], [5].

*Supported in part by NSF Grant CCR9818411 email: alan@random.math.cmu.edu

$\dagger$ Permanent Address Computer and Automation Research Institute of the Hungarian Academy of Sciences, Budapest, P.O.Box 63, Hungary-1518. Supported in part by OTKA Grants T 030059 and T 29074 FKFP 0607/1999. email: ruszinko@lutra.sztaki.hu

${ }^{\ddagger}$ Supported in part by NSF grant DMS-9970622. email: thoma@qwes.math.cmu.edu 
Theorem 1 For any n-vertex connected graph $G$,

$$
\operatorname{mst}(G) \geq \frac{n}{\Delta}\left(\zeta(3)-\epsilon_{1}\right)
$$

where $\Delta=\Delta(G)$ denotes the maximum degree in $G$ and $\epsilon_{1}=\epsilon_{1}(\Delta) \rightarrow 0$ as $\Delta \rightarrow \infty$.

For an upper bound we need expansion properties of $G$.

Theorem 2 Let $\alpha=\alpha(r)=O\left(r^{-1 / 3}\right)$ and let $\rho=\rho(r)$ and $\omega=\omega(r)$ tend to infinity with $r$. Suppose that the graph $G=(V, E)$ is connected and satisfies

$$
r \leq \delta \leq \Delta \leq(1+\alpha) r
$$

where $\delta=\delta(G)$ denotes the minimum degree in $G$. Suppose also that

$$
|(S: \bar{S})| /|S| \geq \omega r^{2 / 3} \log r \text { for all } S \subseteq V \text { with } r / 2<|S| \leq \min \{\rho r,|V| / 2\},
$$

where $(S: \bar{S})=\{(x, y) \in E: x \in S, y \in \bar{S}=E \backslash S\}$. Then

$$
\left|\operatorname{mst}(G)-\frac{n}{r} \zeta(3)\right| \leq \epsilon_{2} \frac{n}{r}
$$

where the $\epsilon_{2}=\epsilon_{2}(r) \rightarrow 0$ as $r \rightarrow \infty$.

For regular graphs we of course take $\alpha=0$.

The expansion condition in the above theorem is probably not the "right one" for obtaining $\operatorname{mst}(G) \sim \frac{n}{r} \zeta(3)$. We conjecture that high edge connectivity is sufficient: Let $\lambda=\lambda(G)$ denote the edge connectivity of $G$.

\section{Conjecture 1}

Suppose that (1) holds. Then,

$$
\left|m s t(G)-\frac{n}{r} \zeta(3)\right| \leq \epsilon_{3} \frac{n}{r}
$$

where $\epsilon_{3}=\epsilon_{3}(\lambda) \rightarrow 0$ as $\lambda \rightarrow \infty$.

Note that $\lambda \rightarrow \infty$ implies $r \rightarrow \infty$.

Along these lines, we prove the following theorem.

Theorem 3 Assume $\alpha=\alpha(r)=O\left(r^{-1 / 3}\right)$ and (1) is satisfied. Suppose that $r \geq \lambda(G) \geq$ $\omega r^{2 / 3} \log n$ where $\omega=\omega(r)$ tends to infinity with $r$. Then

$$
\left|\operatorname{mst}(G)-\frac{n}{r} \zeta(3)\right| \leq \epsilon_{4} \frac{n}{r}
$$

where the $\epsilon_{4}=\epsilon_{4}(r) \rightarrow 0$ as $r \rightarrow \infty$. 
Remark: It is worth pointing out that it is not enough to have $r \rightarrow \infty$ in order to have the result of Theorem 2, that is, we need some extra condition such as high edge connectivity. For consider the graph $\Gamma(n, r)$ obtained from $n / r$-cliques $C_{1}, C_{2}, \ldots, C_{n / r}$ by deleting an edge $\left(x_{i}, y_{i}\right)$ from $C_{i}, 1 \leq i \leq n / r$ then joining the cliques into a cycle of cliques by adding edges $\left(y_{i}, x_{i+1}\right)$ for $1 \leq i \leq n / r$. It is not hard to see that

$$
\operatorname{mst}(\Gamma(n, r)) \sim \frac{n}{r}\left(\zeta(3)+\frac{1}{2}\right)
$$

if $r \rightarrow \infty$ with $r=o(n)$. We repeat the conjecture from [1] that this is the worst-case, i.e.

Conjecture 2 Assuming only the conditions of Theorem 1,

$$
m s t(G) \leq \frac{n}{\delta}\left(\zeta(3)+\frac{1}{2}+\epsilon_{5}\right)
$$

where $\epsilon_{5}=\epsilon_{5}(\delta) \rightarrow 0$ as $\delta \rightarrow \infty$.

We prove instead

Theorem 4 If $G$ is a connected graph then

$$
\operatorname{mst}(G) \leq \frac{n}{\delta}\left(\zeta(3)+1+\epsilon_{6}\right)
$$

where the $\epsilon_{6}=\epsilon_{6}(\delta) \rightarrow 0$ as $\delta \rightarrow \infty$.

We finally note that high connectivity is not necessary to obtain the result of Theorem 2 . Since if $r=o(n)$ then one can tolerate a few small cuts. For example, let $G$ be a graph which satisfies the conditions of Theorem 2 and suppose $r=o(n)$. Then taking 2 disjoint copies of $G$ and adding a single edge joining them we obtain a graph $G^{\prime}$ for which $m s t\left(G^{\prime}\right) \sim$ $\frac{1}{2}+\frac{n^{\prime}}{r} \zeta(3) \sim \frac{n^{\prime}}{r} \zeta(3)$ where $n^{\prime}=2 n$ is the number of vertices of $G^{\prime}$.

\section{Proof of Theorem 3}

Given a connected graph $G=(V, E)$ with $|V|=n$ and $0 \leq p \leq 1$, let $G_{p}$ be the random subgraph of $G$ with the same vertex set which contains those edges $e$ with $X_{e} \leq p$. Let $\kappa(G)$ denote the number of components of $G$. We shall first give a rather precise description of $\operatorname{mst}(G)$.

\section{Lemma 1 [1]}

For any connected graph $G$,

$$
\operatorname{mst}(G)=\int_{p=0}^{1} \mathbf{E}\left(\kappa\left(G_{p}\right)\right) d p-1 .
$$


We substitute $p=x / r$ in (3) to obtain

$$
\operatorname{mst}(G)=\frac{1}{r} \int_{x=0}^{r} \mathbf{E}\left(\kappa\left(G_{x / r}\right)\right) d x-1 .
$$

Now let $C_{k, x}$ denote the total number of components in $G_{x / r}$ with $k$ vertices. Thus

$$
\operatorname{mst}(G)=\frac{1}{r} \int_{x=0}^{r} \sum_{k=1}^{n} \mathbf{E}\left(C_{k, x}\right) d x-1 .
$$

\section{Proof of Theorem 3}

In order to use (4) we need to consider three separate ranges for $x$ and $k$, two of which are satisfactorily dealt with in [1]. Let $A=(r / \omega)^{1 / 3}, B=\left\lfloor(A r)^{1 / 4}\right\rfloor$ so that each of $B \alpha$, $A B^{2} / r$ and $A / B \rightarrow 0$ as $r \rightarrow \infty$. These latter conditions are needed for the analysis of the first two ranges.

Range 1: $0 \leq x \leq A$ and $1 \leq k \leq B-$ see [1].

$$
\frac{1}{r} \int_{x=0}^{A} \sum_{k=1}^{B} \mathbf{E}\left(C_{k, x}\right) d x \leq(1+o(1)) \frac{n}{r} \zeta(3) .
$$

Range 2: $0 \leq x \leq A$ and $k>B-$ see [1].

$$
\frac{1}{r} \int_{x=0}^{A} \sum_{k=B}^{n} \mathbf{E}\left(C_{k, x}\right) d x=o(n / r) .
$$

Range 3: $x \geq A$.

We use a result of Karger [4]. A cut $(S: \bar{S})=\{(u, v) \in E: u \in S, v \notin S\}$ of $G$ is $\gamma$-minimal if $|(S: \bar{S})| \leq \gamma \lambda$. Karger proved that the number of $\gamma$-minimal cuts is $O\left(n^{2 \gamma}\right)$. We can associate each component of $G_{p}$ with a cut of $G$. Thus

$$
\begin{aligned}
\sum_{k=1}^{n} \mathbf{E}\left(C_{k, x}\right) \leq O\left(\sum_{s=\lambda}^{\infty} n^{2 s / \lambda}\left(1-\frac{x}{r}\right)^{s}\right) & =O\left(\sum_{s=\lambda}^{\infty}\left(n^{2 r / \lambda} e^{-x}\right)^{s / r}\right) \\
= & O\left(\int_{s=\lambda}^{\infty}\left(n^{2 r / \lambda} e^{-x}\right)^{s / r} d s\right)=O\left(\frac{r n^{2} e^{-x \lambda / r}}{x-\frac{2 r}{\lambda} \log n}\right),
\end{aligned}
$$

and using $A \lambda \geq \omega^{2 / 3} r \log n$ we obtain

$$
\begin{aligned}
& \frac{1}{r} \int_{x=A}^{r} \sum_{k=1}^{n} \mathbf{E}\left(C_{k, x}\right) d x=O(\left.\int_{x=A}^{r} \frac{n^{2} e^{-x \lambda / r}}{x-\frac{2 r}{\lambda} \log n} d x\right) \\
&=O\left(A^{-1} \int_{x=A}^{r} n^{2} e^{-x \lambda / r} d x\right)=O\left(\frac{r n^{2}}{A \lambda} e^{-A \lambda / r}\right)=o(n / r) .
\end{aligned}
$$

We complete the proof by applying Lemma 1 . 


\section{Proof of Theorem 4}

We keep the definitions of $A, B$ and Ranges 1,2 , but we split Range 3 and let $\delta=r$.

Range 3a: $x \geq A$ and $k \leq(1-\epsilon) r, 0<\epsilon<1$, arbitrary - see [1] (here $\epsilon=1 / 2$ but the argument works for arbitrary $\epsilon$ ).

$$
\frac{1}{r} \int_{x=A}^{r} \sum_{k=1}^{(1-\epsilon) r} \mathbf{E}\left(C_{k, x}\right) d x=o(n / r) .
$$

Range 3b: $x \geq A$ and $k>(1-\epsilon) r$.

Clearly

$$
\sum_{k=(1-\epsilon) r}^{n} C_{k, x} \leq \frac{n}{(1-\epsilon) r}
$$

and hence

$$
\frac{1}{r} \int_{x=A}^{r} \sum_{k=(1-\epsilon) r}^{n} \mathbf{E}\left(C_{k, x}\right) d x \leq \frac{n}{(1-\epsilon) r}
$$

We again complete the proof by applying Lemma 1.

\section{References}

[1] A. Beveridge, A. M. Frieze and C. J. H. McDiarmid, Minimum length spanning trees in regular graphs, Combinatorica 18 (1998) 311-333.

[2] A. M. Frieze, On the value of a random minimum spanning tree problem, Discrete Applied Mathematics 10 (1985) 47 - 56.

[3] A. M. Frieze and C. J. H. McDiarmid, On random minimum length spanning trees, Combinatorica 9 (1989) 363 - 374.

[4] D. R. Karger, A Randomized Fully Polynomial Time Approximation Scheme for the All Terminal Network Reliability Problem, Proceedings of the twenty-seventh annual ACM Symposium on Theory of Computing (1995) 11-17.

[5] M. Penrose, Random minimum spanning tree and percolation on the $n$-cube, Random Structures and Algorithms 12 (1998) 63 - 82. 